\title{
Full-Duplex Wireless Communications Using Off-The-Shelf Radios: Feasibility and First Results
}

\author{
Melissa Duarte and Ashutosh Sabharwal \\ Department of Electrical and Computer Engineering, Rice University, Houston, TX 77005 \\ Email: \{mduarte, ashu\}@ rice.edu
}

\begin{abstract}
We study full-duplex wireless communication systems where same band simultaneous bidirectional communication is achieved via cancellation of the self-interfering signal. Using offthe-shelf MIMO radios, we present experimental results that characterize the suppression performance of three self-interference cancellation mechanisms, which combine a different mix of analog and digital cancellation. Our experimental results show that while the amount of self-interference increases linearly with the transmitted power, the self-interference can be sufficiently cancelled to make full-duplex wireless communication feasible in many cases. Our experimental results further show that if the self-interference is cancelled in the analog domain before the interfering signal reaches the receiver front end, then the resulting full-duplex system can achieve rates higher than the rates achieved by a half-duplex system with identical analog resources.
\end{abstract}

\section{INTRODUCTION}

Current deployed wireless communication systems employ either a time-division or frequency-division approach to bidirectional communication. This requires dividing the temporal and/or spectral resources into orthogonal resources and results in half-duplex wireless communication systems. This paper studies full-duplex wireless communication systems which consist on same band and simultaneous bidirectional communication.

We consider a system where two nodes communicate to each other in full-duplex mode and each node has two antennas, one antenna is used for transmission and the other antenna is used for reception. Antenna Separation is the simplest passive self-interference cancellation mechanism and it consists in loss in interference power due to propagation losses caused by separating the transmit and receive antennas at a node. The more separation between interfering antennas the better, but this will have an impact on the size of the wireless nodes. For our experiments we have considered distances of $20 \mathrm{~cm}$ and $40 \mathrm{~cm}$ between interfering antennas. These are feasible separations for mobile devices like laptops but the separation is not enough to attenuate the interfering signal such that the interference power is below the power of the signal of interest. Hence, we use Analog Cancellation and Digital Cancellation in order to achieve larger attenuation of the self-interfering signal.

Analog Cancellation is an active cancellation mechanism which sends a canceling signal through another radio chain and adds it to the signal at the receiving antenna. Digital Cancellation is an active cancellation mechanism which uses the knowledge of the interfering signal to cancel the interfering signal in baseband. In our prototype implementation we consider a combination of Antenna Separation, Analog Cancellation, and Digital Cancellation. More specifically, we have implemented the following three mechanisms for selfinterference cancellation. 1) Antenna Separation and Digital Cancellation, 2) Antenna Separation and Analog Cancellation, and 3) Antenna Separation, Analog and Digital Cancellation. We present an experimental evaluation of these three different self-interference cancellation mechanisms and their effect on the performance of full-duplex wireless systems. For our experiments we have considered transmission powers from $-5 \mathrm{dBm}$ to $15 \mathrm{dBm}$, distances of $20 \mathrm{~cm}$ and $40 \mathrm{~cm}$ between interfering antennas, and a distance of $6.5 \mathrm{~m}$ between nodes. Hence, our study considers transmission powers and distances between nodes and interfering antennas that are typical to Bluetooth and WiFi systems.

Our first contribution is a measurement based characterization of the amount of cancellation achieved by the three selfinterference cancellation mechanisms. We show that the selfinterfering signal can be suppressed by more than $70 \mathrm{~dB}$. Further, the power of the interfering signal after cancellation can be well approximated as a linear function of the transmission power of the interfering antenna. The average percentage error between the measured data and the data fitted by the linear approximation is always less than $3 \%$ for all our measurements.

Our second contribution is a comparison of the achievable rates of full-duplex and half-duplex systems based on overthe-air wireless experiments. Our experimental results show that the self-interference cancellation mechanisms that we have considered can cancel interference so that full-duplex wireless communication is feasible. Furthermore, our results show that if the self-interference is canceled before the interfering signal reaches the receiver front end, then the achievable rate of the full-duplex system can be higher then the achievable rate of a half-duplex system which uses the same amount of resources.

Previous work on measurement based characterization of self-interference cancellation mechanisms has been presented in [1-3]. Work in [1,3] characterizes self-interference cancellation mechanisms that are based on multiple antenna techniques and require more than two antennas per full-duplex node. Work in [2] characterizes self-interference cancellation mechanisms that take advantage of antenna directionality. The selfinterference cancellation mechanisms that we have considered correspond to the case where there is a constraint of only two antennas per full-duplex node and we use typical WiFi antennas which provide less interference isolation than the directional 


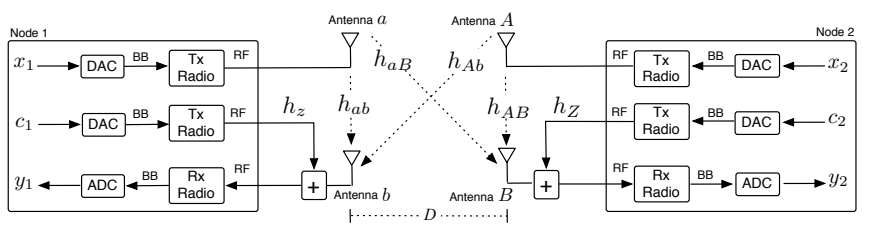

Fig. 1. RF model of a full-duplex system.

antennas used in [2]. Hence, compared to the work in [1-3], we have considered self-interference scenarios that are more constrained in terms of the resources that are available for self-interference cancellation but still correspond to realistic scenarios.

Work in $[4,5]$ shows characterization of self-interference cancellation mechanisms for scenarios similar to the ones we have considered. However, although work in $[4,5]$ reports the amount of self-interference cancellation that can be achieved by their proposed system, they do not report if the resulting full-duplex system can achieve better performance than a halfduplex system. We provide comparison of achievable rates of full-duplex and half-duplex systems for all the self-interference cancellation mechanisms that we have considered. A similar comparison is provided in [6] but the authors have analyzed full-duplex systems with center frequencies and transmission powers different from the ones we have considered. The fullduplex system presented in [6] has center frequencies of around $600 \mathrm{MHz}$ while the full-duplex systems we analyze have a center frequency of $2.4 \mathrm{GHz}$. Center frequencies of $2.4 \mathrm{GHz}$ are used by $\mathrm{WiFi}$ and Bluetooth systems and result in fullduplex systems with higher self-interference because higher transmission powers are required as pointed out in [6].

\section{MEChanisms For SELF InTERFEREnCE CANCELLATION}

The RF model of the full-duplex system considered is shown in Fig. 1. We use $x_{1}$ to denote the signal transmitted from Node 1 (N1), $h_{a B}$ to denote the wireless channel from antenna $a$ to antenna $B$, and $h_{a b}$ to denote the wireless channel from antenna $a$ to antenna $b$. Similarly we use $x_{2}$ to denote the signal transmitted from Node $2(\mathrm{~N} 2), h_{A b}$ to denote the wireless channel from antenna $A$ to antenna $b$, and $h_{A B}$ to denote the wireless channel from antenna $A$ to antenna $B$. For our experiments signals $x_{1}$ and $x_{2}$ are narrowband with a bandwidth of $625 \mathrm{kHz}$ (we are currently working on extending our results to systems with wider bandwidths). We label $d$ as the distance between interfering antennas (same node antennas) and $D$ as the distance between nodes. For our experiments we have considered $d=20$ $\mathrm{cm}$ and $d=40 \mathrm{~cm}$ of separation between same node antennas and and a fixed distance $D=6.5 \mathrm{~m}$ between nodes. The three self-interference cancellation mechanisms that we analyze are explained below

1) Antenna Separation and Digital Cancellation (ASDC): If only antenna separation is used for self-interference cancellation then the self-interfering signal at $\mathrm{N} 1$ is equal to $h_{a b} x_{1}$ and the self-interfering signal at $\mathrm{N} 2$ is equal to $h_{A B} x_{2}$.
$\mathrm{N} 1$ and $\mathrm{N} 2$ can estimate $h_{a b}$ and $h_{A B}$ respectively and use these estimates in the digital domain to further cancel the interference by subtracting $\widehat{h}_{a b} x_{1}$ at N1 and $\widehat{h}_{A B} x_{2}$ at N2 from the received signal. We use $\widehat{h}_{a b}$ to denote the noisy estimate of $h_{a b}$ and $\widehat{h}_{A B}$ to denote the noisy estimate of $h_{A B}$. After applying Antenna Separation and Digital Cancellation (ASDC), the interfering signal at N1 is equal to $\left(h_{a b}-\widehat{h}_{a b}\right) x_{1}$ and the interfering signal at $\mathrm{N} 2$ is equal to $\left(h_{A B}-\widehat{h}_{A B}\right) x_{2}$. Perfect cancellation is not possible due to error in the estimation of $h_{a b}$ and $h_{A B}$. The power of the interfering signal at N1 and $\mathrm{N} 2$ after ASDC is given by $\mathrm{P}_{\mathrm{ASDC}}^{\mathrm{N} 1}=E\left[\left|\left(h_{a, b}-\widehat{h}_{a, b}\right) x_{1}\right|^{2}\right]$ and $\mathrm{P}_{\mathrm{ASDC}}^{\mathrm{N} 2}=E\left[\left|\left(h_{A, B}-\widehat{h}_{A, B}\right) x_{2}\right|^{2}\right]$ respectively.

Because ASDC cancels the interference in the digital domain two problems may arise. First, for small antenna separation the magnitude of the interfering signal can be large enough to saturate the receiver front end. Second, even if there is no receiver front end saturation, we have that at the input of the analog to digital converter the magnitude of the interfering signal is larger than the magnitude of the signal of interest and this results in quantization noise. This quantization noise will not be reduced by the digital self-interference cancellation. Using self-interference cancellation in the analog domain can help mitigate the two problems mentioned above, hence, we have also considered two self-interference cancellation mechanisms that use analog cancellation. These two mechanisms are explained below

2) Antenna Separation and Analog Cancellation (ASAC): The analog self-interference canceller for N1 consists in sending the canceller signal $c_{1}$ through an additional transmitter radio that converts the signal to $\mathrm{RF}$ and adding the output of the radio to the received signal. We use $h_{z}$ to denote the magnitude and phase applied by N1's transmitter RF chain to signal $c_{1}$. One can easily see that in order to cancel the self-interference at $\mathrm{N} 1$ the canceller signal must be equal to $c_{1}=-\left(h_{a b} / h_{z}\right) x_{1}$. However, since there is additive noise and other distortions in the system, N1 cannot have a perfect estimate of channels $h_{a b}$ and $h_{z}$. Consequently, the analog canceller cannot completely cancel the self-interference. We use $\widehat{h}_{z}$ to denote the noisy estimate of $h_{z}$. The interfering signal at N1 after Antenna Separation and Analog Cancellation (ASAC) is equal to $\left(h_{a b}-h_{z} \widehat{h}_{a b} / \widehat{h}_{z}\right) x_{1}$. The power of the interfering signal at $\mathrm{N} 1$ after ASAC is given by $\mathrm{P}_{\mathrm{ASAC}}^{\mathrm{N} 1}=E\left[\left|\left(h_{a b}-h_{z} \widehat{h}_{a b} / \widehat{h}_{z}\right) x_{1}\right|^{2}\right]$.

Following a similar analysis as above we obtain that the self-interfering signal at $\mathrm{N} 2$ after ASAC is equal to $\left(h_{A B}-\right.$ $\left.h_{Z} \widehat{h}_{A B} / \widehat{h}_{Z}\right) x_{2}$ where we use $h_{Z}$ to denote the magnitude and phase applied by N2's transmitter RF chain to signal $c_{2}$. This signal is given by $c_{2}=-\left(\widehat{h}_{A B} / \widehat{h}_{Z}\right) x_{2}$ and $\widehat{h}_{Z}$ denotes the noisy estimate of $h_{Z}$. The power of the interfering signal at N2 after ASAC is given by $\mathrm{P}_{\mathrm{ASAC}}^{\mathrm{N} 2}=E\left[\left|\left(h_{A B}-h_{Z} \widehat{h}_{A B} / \widehat{h}_{Z}\right) x_{2}\right|^{2}\right]$.

3) Antenna Separation, Analog and Digital Cancellation $(A S A D C)$ : The ASAC mechanism cannot completely cancel the self-interference due to noise in the estimate of the signals required for cancellation. Hence, we consider the use of digital cancellation after ASAC to achieve larger cancellation of the self-interfering signal. We define $h_{\mathrm{ASAC}}^{\mathrm{N} 1}=h_{a b}-h_{z} \widehat{h}_{a b} / \widehat{h}_{z}$ 
and $h_{\mathrm{ASAC}}^{\mathrm{N} 2}=h_{A B}-h_{Z} \widehat{h}_{A B} / \widehat{h}_{Z} \cdot h_{\mathrm{ASAC}}^{\mathrm{N} 1}$ and $h_{\mathrm{ASAC}}^{\mathrm{N} 2}$ are the equivalent self-interfering channel after ASAC at N1 and N2 respectively. The digital cancellation after ASAC consists in estimating $h_{\mathrm{ASAC}}^{N 1}$ and $h_{\mathrm{ASAC}}^{\mathrm{N} 2}$ and using these estimates to cancel the interfering signal in the digital domain. We use $\widehat{h}_{\mathrm{ASAC}}^{\mathrm{N} 1}$ to denote the noisy estimate of $h_{\mathrm{ASAC}}^{\mathrm{N} 1}$ and we use $\widehat{h}_{\mathrm{ASAC}}^{\mathrm{N} 2}$ to denote the noisy estimate of $h_{\mathrm{ASAC}}^{\mathrm{N} 2}$.

The self-interfering signal after Antenna Separation, Analog, and Digital Cancellation (ASADC) at $\mathrm{N} 1$ is given by $\left(h_{\mathrm{ASAC}}^{\mathrm{N} 1}-\right.$ $\left.\widehat{h}_{\mathrm{ASAC}}^{\mathrm{N} 1}\right) x_{1}$ and the self-interfering signal after ASADC at N2 is given by $\left(h_{\mathrm{ASAC}}^{\mathrm{N} 2}-\widehat{h}_{\mathrm{ASAC}}^{\mathrm{N} 2}\right) x_{2}$. The power of the self-interfering signal at $\mathrm{N} 1$ after ASADC is given by $\mathrm{P}_{\mathrm{ASADC}}^{\mathrm{N} 1}=E\left[\mid\left(h_{\mathrm{ASAC}}^{\mathrm{N} 1}-\right.\right.$ $\left.\left.\widehat{h}_{\mathrm{ASAC}}^{\mathrm{N} 1}\right)\left.x_{1}\right|^{2}\right]$. The power of the self-interfering signal at $\mathrm{N} 2$ after $\mathrm{ASADC}$ is given by $\mathrm{P}_{\mathrm{ASADC}}^{\mathrm{N} 2}=E\left[\left|\left(h_{\mathrm{ASAC}}^{\mathrm{N} 2}-\widehat{h}_{\mathrm{ASAC}}^{\mathrm{N} 2}\right) x_{2}\right|^{2}\right]$.

\section{Measurement Based Characterization}

In this section we present a measurement based characterization of the amount of cancellation that can be achieved by each of the three self-interference cancellation mechanisms described in the previous section. We used the WARPLab framework [7] to implement the full-duplex system shown in Fig. 1. The RF signals were transmitted and received over-the-air in real time and digital baseband signals were processed offline using MATLAB. We used typical WiFi antennas [8].

Fig. 2(a) and Fig. 2(b) show measurements of the power of the interfering signal after applying different cancellation mechanisms. Results in Fig. 2(a) correspond to measurements made at $\mathrm{N} 1$ for a distance of $20 \mathrm{~cm}$ between interfering antennas. Results in Figure 2(b) correspond to measurements made at N2 for a distance of $40 \mathrm{~cm}$ between interfering antennas. Fig. 2(a) and Fig. 2(b) also show the measured power of the of the signal of interest at $\mathrm{N} 1$ and $\mathrm{N} 2$ respectively. The power of the signal of interest at $\mathrm{N} 1$ is given by $\mathrm{P}_{\mathrm{N} 2 \mathrm{~N} 1}=E\left[\left|h_{A b} x_{2}\right|^{2}\right]$ and the power of the signal of interest at $\mathrm{N} 2$ is given by $\mathrm{P}_{\mathrm{N} 1 \mathrm{~N} 2}=E\left[\left|h_{a B} x_{1}\right|^{2}\right]$. All our results correspond to a node separation of $D=6.5 \mathrm{~m}$. The x-axis in Fig. 2(a) and Fig. 2(b) is the transmitted power. We consider a symmetric system hence N1 and N2 transmit with the same power given by $\mathrm{P}_{\mathrm{TX}}=E\left[\left|x_{1}\right|^{2}\right]=E\left[\left|x_{2}\right|^{2}\right]$. For our experiments we have considered transmission powers from $-5 \mathrm{dBm}$ to $15 \mathrm{dBm}$. For each transmission power and cancellation mechanism considered we performed a total of 1250 measurements over a time span of 9 hours; the plots in Fig. 2(a) and Fig. 2(b) were obtained by averaging these measurements.

Since the three self-interference cancellation mechanisms that we have considered use Antenna Separation (AS) as part of the cancellation mechanism, we first characterize the amount of cancellation achieved by AS only. The power of the interfering signal at $\mathrm{N} 1$ after $\mathrm{AS}$ is equal to $\mathrm{P}_{\mathrm{AS}}^{\mathrm{N} 1}=E\left[\left|h_{a b} x_{1}\right|^{2}\right]$ and the power of the interfering signal at $\mathrm{N} 2$ after $\mathrm{AS}$ is equal to $\mathrm{P}_{\mathrm{AS}}^{\mathrm{N} 2}=E\left[\left|h_{A B} x_{2}\right|^{2}\right]$. From Fig. 2(a) and Fig. 2(b) we observe that AS only is not enough to reduce the power of the interfering signal to levels below the power of the signal of interest, however, this can be achieved using either ASDC, ASAC, or ASADC. We observe that ASDC and ASAC can achieve similar amounts of cancellation and ASADC is the cancellation mechanism that achieves the largest cancellation out of the mechanisms considered.

Our measurements also show that, for a fixed distance between interfering antennas, the amount of cancellation achieved by a given mechanism remains approximately constant over all the transmission powers considered. The average amount of cancellation (averaged over all measurements at different nodes and different transmit powers for a fixed distance between interfering antennas) achieved by AS, ASDC, ASAC, and ASADC is shown in Table I. We observe that a using the values in Table I we can obtain a good approximation of the power of the interfering signal for a given cancellation mechanism. For all four cancellation mechanisms (AS, ASAC, ASAC and ASADC), the power of the self-interfering signal can be well approximated as a linear function of the transmitted power. In $\mathrm{dBm}$, this approximation is equal to the transmission power in $\mathrm{dBm}$ minus the average attenuation in $\mathrm{dB}$, where the average attenuation for each mechanism and each distance between interfering antennas considered is shown in Table I. Plots of the approximations are shown in Fig. 2(a) and Fig. 2(b), we observe that the linear approximation of the self-interference power is a good estimate for all the measurements. Table II shows the average percentage error for the approximations, from this table we observe that the average percentage error was always less than $3 \%$ for all our measurements.

TABLE I

AVERAGE AMOUNT OF CANCELLATION ACHIEVED BY DIFFERENT SELF-INTERFERENCE CANCELLATION MECHANISMS AT 20 CM AND 40 CM SPACING BETWEEN INTERFERING ANTENNAS

\begin{tabular}{|c|c|c|c|c|}
\hline & AS & ASDC & ASAC & ASADC \\
\hline \hline $20 \mathrm{~cm}$ & $39 \mathrm{~dB}$ & $70 \mathrm{~dB}$ & $72 \mathrm{~dB}$ & $78 \mathrm{~dB}$ \\
\hline $40 \mathrm{~cm}$ & $45 \mathrm{~dB}$ & $76 \mathrm{~dB}$ & $76 \mathrm{~dB}$ & $80 \mathrm{~dB}$ \\
\hline
\end{tabular}

TABLE II

PERCENTAGE ERROR FOR THE LINEAR APPROXIMATION FOR CHARACTERIZATION OF THE INTERFERENCE POWER AFTER CANCELLATION. RESULTS ARE GIVEN FOR THE DIFFERENT SELF-INTERFERENCE CANCELLATION MECHANISMS CONSIDERED AND FOR DISTANCES OF $20 \mathrm{CM}$ AND $40 \mathrm{CM}$ BETWEEN INTERFERING ANTENNAS.

\begin{tabular}{|c|c|c|c|c|}
\hline & AS & ASDC & ASAC & ASADC \\
\hline \hline $20 \mathrm{~cm}$ & $2.0 \%$ & $1.4 \%$ & $3.0 \%$ & $1.9 \%$ \\
\hline $40 \mathrm{~cm}$ & $2.2 \%$ & $2.8 \%$ & $1.7 \%$ & $2.6 \%$ \\
\hline
\end{tabular}

From Fig. 2(a), Fig. 2(b), and Table I, we observe that adding digital cancellation to AS increased the total amount of cancellation by approximately $31 \mathrm{~dB}$. For example, we can see from Table I that at $20 \mathrm{~cm}$ separation between interfering antennas the average cancellation increased from $39 \mathrm{~dB}$ to $70 \mathrm{~dB}$. Notice that adding digital cancellation to ASAC did not not achieve such a large increase in cancellation. Observe from Table I that at $20 \mathrm{~cm}$ separation between interfering antennas 


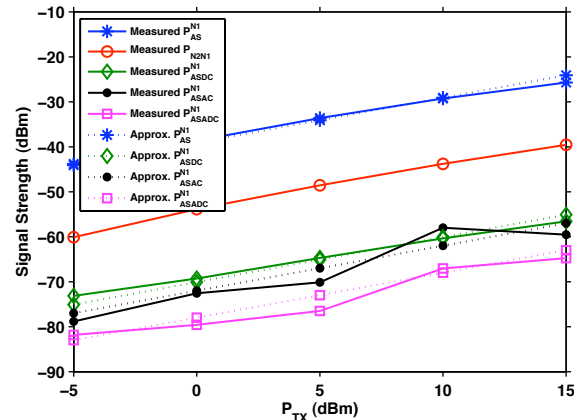

(a) Measured and approximated signal powers at N1 with $d=20 \mathrm{~cm}$.

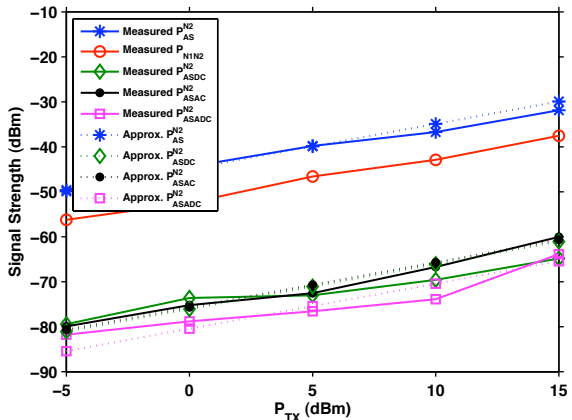

(b) Measured and approximated signal powers at $\mathrm{N} 2$ with $d=40 \mathrm{~cm}$.

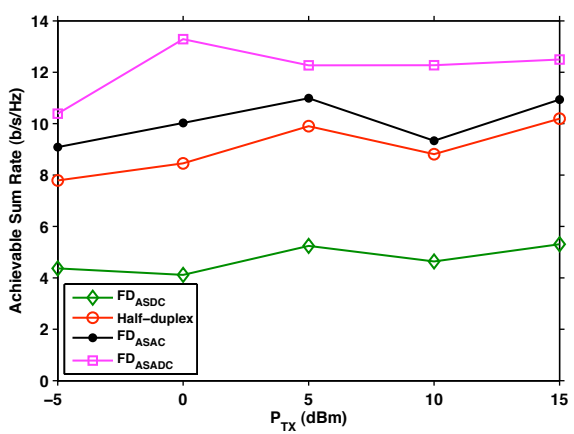

(c) Achievable sum rates for $d=20 \mathrm{~cm}$ and $D=$ $6.5 \mathrm{~m}$.

Fig. 2. Experiment Results.

the average cancellation for ASAC was $72 \mathrm{~dB}$ and adding digital cancellation increased the attenuation by approximately $6 \mathrm{~dB}$ to obtain a total of $78 \mathrm{~dB}$ of cancellation for the ASADC mechanism. Our intuition behind these results is the following. From Table I, Fig. 2(a), and Fig. 2(b), we observe that for the ASDC mechanism the power of the interfering signal before digital cancellation is stronger (approximately $32 \mathrm{~dB}$ stronger as can be computed from results in Table I by subtracting results for ASAC from results for AS) than the power of the interfering signal before digital cancellation in the ASADC mechanism. To apply digital cancellation we need an estimate of the interfering channel and this estimate is obtained from the interfering signal. We conjecture that, because the interfering signal before digital cancellation is much stronger in the ASDC mechanism than in the ASADC mechanism, the ASDC mechanism is able to get a better estimate of the interfering channel before digital cancellation than the ASADC mechanism. This better quality in the channel estimate results in a larger amount of cancellation achieved in the digital domain.

\section{Full-Duplex versus Half-Duplex Achievable RATES}

Results in Table I show that it is possible to reduce the selfinterference by more than $70 \mathrm{~dB}$. We are now interested in understanding if the self- interference cancellation mechanisms that we have described and analyzed can result in full-duplex systems that have better performance than half-duplex systems. As we will show in this section, it is feasible to implement full-duplex systems that achieve better performance than halfduplex systems, hence, full-duplex is a feasible and interesting option for future wireless communications systems.

We have used the WARPLab framework to implement a full-duplex system that uses ASDC, a full-duplex system that used ASAC, and a full-duplex system that uses ASADC. We label these three systems as $\mathrm{FD}_{\mathrm{ASDC}}, \mathrm{FD}_{\mathrm{ASAC}}$, and $\mathrm{FD}_{\mathrm{ASADC}}$ respectively. For fair comparison between full-duplex and halfduplex systems we have considered a half-duplex Alamouti system with two transmitter antennas and one receiver antenna. This half-duplex system uses two transmit RF chains per node and one receive RF chain per node, which is the same number of RF chains per node available in the full-duplex implementation as shown in Fig. 1.

The half-duplex experiments were implemented using the WARPLab framework and used the same distance between nodes and same distance between antennas at a node as considered in the full-duplex experiments. For the half-duplex experiments we first transmit from N1 to $\mathrm{N} 2$ and we use both antennas at $\mathrm{N} 1$ for transmission and the antenna connected to the single RF chain at $\mathrm{N} 2$ for reception. We then transmit from $\mathrm{N} 2$ to $\mathrm{N} 1$ and we use both antennas at $\mathrm{N} 2$ for transmission and the antenna connected to the single RF chain at N1 for reception. We normalize the total transmitted power of the full-duplex and half-duplex systems considered by fixing the total power transmitted per antenna equal to $\mathrm{P}_{\mathrm{TX}}$. Note that for all the systems considered there are always two antennas transmitting a signal. Hence, all the systems considered have the same average transmitted power when we take into account the power transmitted by both nodes.

We compare the half-duplex and full-duplex systems by comparing their achievable sum rates. We estimate achievable rates based on the SINR per frame which is computed based on the measured Average Error Vector Magnitude Squared (AEVMS) [9] per frame. The AEVMS per frame is measured as follows. We transmit a normalized constellation symbol $s$ and compute its estimate $\widehat{s}$ at the receiver, $\widehat{s}$ is the decision variable that is input to the demodulator. The AEVMS per frame is given by AEVMS $=E\left[|s-\widehat{s}|^{2}\right]$ where the expectation is over all symbols transmitted during a frame. In a half-duplex system the estimate $\widehat{s}$ is different from $s$ due to noise at the receiver and in a full-duplex system $\widehat{s}$ is different from $s$ due to both self-interference and noise at the receiver. We estimate the SINR per frame as SINR $=1 /$ AEVMS, notice the numerator in the SINR expression is equal to one because we are using a normalized constellation. The achievable rate per frame is computed as $\log (1+$ SINR $)$.

Table III shows the equations that were used for computation of the achievable rates. We use $F$ to denote the total number of frames. For our experiments we transmitted a total of 1250 frames per system and per transmission power considered, the 1250 frames were transmitted over a time span of 9 hours. 
We use $\operatorname{SINR}\left[f, \mathrm{~N} 1 \mathrm{~N} 2, \mathrm{FD}_{\mathrm{ASDC}}\right], \quad \operatorname{SINR}\left[f, \mathrm{~N} 1 \mathrm{~N} 2, \mathrm{FD}_{\mathrm{ASAC}}\right]$, $\operatorname{SINR}\left[f, \mathrm{~N} 1 \mathrm{~N} 2, \mathrm{FD}_{\mathrm{ASADC}}\right]$, and $\operatorname{SINR}[f, \mathrm{~N} 1 \mathrm{~N} 2, \mathrm{HD}]$ to denote the SINR for the $f$-th frame transmitted from N1 to N2 for the $\mathrm{FD}_{\mathrm{ASDC}}, \mathrm{FD}_{\mathrm{ASAC}}, \mathrm{FD}_{\mathrm{ASADC}}$, and half-duplex system respectively. Similarly, we use $\operatorname{SINR}\left[f, \mathrm{~N} 2 \mathrm{~N} 1, \mathrm{FD}_{\mathrm{ASDC}}\right]$, $\operatorname{SINR}\left[f, \mathrm{~N} 2 \mathrm{~N} 1, \mathrm{FD}_{\mathrm{ASAC}}\right], \quad \operatorname{SINR}\left[f, \mathrm{~N} 2 \mathrm{~N} 1, \mathrm{FD}_{\mathrm{ASADC}}\right], \quad$ and $\operatorname{SINR}[f, \mathrm{~N} 2 \mathrm{~N} 1, \mathrm{HD}]$ to denote the SINR for the $f$-th frame transmitted from $\mathrm{N} 2$ to $\mathrm{N} 1$ for the $\mathrm{FD}_{\mathrm{ASDC}}, \mathrm{FD}_{\mathrm{ASAC}}, \mathrm{FD}_{\mathrm{ASADC}}$, and half-duplex system respectively. Notice that for the HD system the achievable rate expression has a factor of 0.5 . This is because in HD mode the wireless channel is time shared and $50 \%$ of the time $\mathrm{N} 1$ is transmitting to $\mathrm{N} 2$ and the other $50 \%$ of the time $\mathrm{N} 2$ is transmitting to $\mathrm{N} 1$.

TABLE III

EQUATIONS FOR COMPUTATION OF ACHIEVABLE RATES

\begin{tabular}{|c||c|}
\hline FD $_{\text {ASDC }}$ & $\begin{array}{c}(1 / F) \sum_{f=1}^{F}\left(\log \left(1+\operatorname{SINR}\left[f, \mathrm{~N} 1 \mathrm{~N} 2, \mathrm{FD}_{\mathrm{ASDC}}\right]\right)\right. \\
\left.+\log \left(1+\operatorname{SINR}\left[f, \mathrm{~N} 2 \mathrm{~N} 1, \mathrm{FD}_{\mathrm{ASDC}}\right]\right)\right)\end{array}$ \\
\hline $\mathrm{FD}_{\mathrm{ASAC}}$ & $\begin{array}{c}(1 / F) \sum_{f=1}^{F}\left(\log \left(1+\operatorname{SINR}\left[f, \mathrm{~N} 1 \mathrm{~N} 2, \mathrm{FD}_{\mathrm{ASAC}}\right]\right)\right. \\
\left.+\log \left(1+\operatorname{SINR}\left[f, \mathrm{~N} 2 \mathrm{~N} 1, \mathrm{FD}_{\mathrm{ASAC}}\right]\right)\right)\end{array}$ \\
\hline $\mathrm{FD}_{\mathrm{ASADC}}$ & $\begin{array}{c}(1 / F) \sum_{f=1}^{F}\left(\log \left(1+\operatorname{SINR}\left[f, \mathrm{~N} 1 \mathrm{~N} 2, \mathrm{FD}_{\mathrm{ASADC}}\right]\right)\right. \\
\left.+\log \left(1+\operatorname{SINR}\left[f, \mathrm{~N} 2 \mathrm{~N} 1, \mathrm{FD} \mathrm{ASADC}_{\mathrm{ASA}}\right]\right)\right)\end{array}$ \\
\hline Half-Duplex & $(0.5 / F) \sum_{f=1}^{F}(\log (1+\mathrm{SINR}[f, \mathrm{~N} 1 \mathrm{~N} 2, \mathrm{HD}]$ \\
& $+\log (1+\operatorname{SINR}[f, \mathrm{~N} 2 \mathrm{~N} 1, \mathrm{HD}]))$ \\
\hline
\end{tabular}

Figure 2(c) shows the estimated achievable rates for the four systems considered and for different transmission powers. In the previous section we observed from Fig. 2(a), Fig. 2(b), and Table I that the ASDC and ASAC mechanisms achieved almost the same amount of self-interference cancellation. However, Fig. 2(c) shows that the achievable rate for the $\mathrm{FD}_{\mathrm{ASAC}}$ system is larger than the achievable rate for the $\mathrm{FD}_{\mathrm{ASDC}}$ system. The reason for this behavior is that, although both ASDC and ASAC mechanisms can cancel the same amount of interference, the ASDC mechanism results in larger quantization noise because it cancels the interference only in the digital domain. In other words, the ASAC mechanism results in lower quantization noise because the interfering signal is canceled in the analog domain before it reaches the receiver front end, consequently, at the input of the analog to digital converter the signal of interest is larger than the interfering signal.

Figure 2(c) shows that the $\mathrm{FD}_{\mathrm{ASAC}}$ and $\mathrm{FD}_{\mathrm{ASADC}}$ systems can achieve better performance than the half-duplex system. We conclude that if the self-interference is canceled before the interfering signal reaches the receiver front end then the resulting full-duplex system can have better performance than a half-duplex system that uses the same amount of resources.

Results in Fig. 2(c) correspond to a fixed distance $D=$ 6.5 meters between nodes and distance $d=20 \mathrm{~cm}$ between interfering antennas. As expected and verified in Table I, increasing/decreasing $d$ will decrease/increase the selfinterference. It is expected that increasing/decreasing $D$ will deteriorate/improve the performance of both full-duplex and half-duplex systems. As future work, we would like to derive an equivalent baseband model for the self-interference cancellation and for the full-duplex systems. Having these models will facilitate analytical comparison between full-duplex and half-duplex systems and will also facilitate understanding the tradeoffs as a function of the system parameters. This will facilitate understanding performance of full-duplex systems in a more general way without having to build and test full-duplex and half-duplex systems for comparison under different system parameters.

Among all the tested full-duplex systems, the $\mathrm{FD}_{\mathrm{ASADC}}$ system has the best performance. This result is in agreement with the results obtained in the previous section that showed that the ASADC mechanism achieved the largest cancellation. Although the $\mathrm{FD}_{\mathrm{ASDC}}$ system has the worst performance, the results in Fig. 2(c) show that it is still feasible system since it can achieve rates larger than $1 \mathrm{~b} / \mathrm{s} / \mathrm{Hz}$.

\section{CONCLUSIONS}

We have presented a measurement based characterization of different self-interference cancellation mechanisms. We have analyzed, via experimental results, the effect that different selfinterference cancellation mechanisms have in the performance of full-duplex systems. Our results demonstrate that full-duplex systems are feasible and can achieve rates larger than the rates achieved by half-duplex systems. These results open the possibility for the design of new systems and network protocols based on full-duplex nodes. It has been noted in $[3,6]$ that protocols based on full-duplex nodes can have some advantages over protocols based on half-duplex nodes. For example, fullduplex nodes can help solve the hidden node problem. Also, in a cognitive radio system, a secondary user with full-duplex capabilities can be always listening as it transmits and consequently, it can immediately stop its transmission when it detects the presence of a primary transmission.

\section{REFERENCES}

[1] D. W. Bliss, P. Parker, and A. R. Margetts, "Simultaneous transmission and reception for improved wireless network performance," in Statistical Signal Processing, 2007. SSP '07. IEEE/SP 14th Workshop on, Aug. 2007, pp. 478-482.

[2] K. Haneda, E. Kahra, S. Wyne, C. Icheln, and P. Vainikainen, "Measurement of loop-back interference channels for outdoor-to-indoor fullduplex radio relays," in Proceedings of the fourth European Conference on Antennas and Propagation EUCAP, 2010.

[3] J. I. Choi, M. Jain, K. Srinivasan, P. Levis, and S. Katti, "Achieving single channe, full duplex wireless communication," in MobiCom, 2010.

[4] S.Chen, M. Beach, and J. McGeehan, "Division-free duplex for wireless applications," in IEEE Electronics Letters, vol. 34, no. 2, 1998, pp. 147148 .

[5] A. Raghavan, E. Gebara, E. M. Tentzeris, and J. Laskar, "Analysis and design of an interference canceller for collocated radios," IEEE Transactions on Microwave Theory and Techniques, vol. 53, no. 3498-3508, 2005.

[6] B. Radunovic, D. Gunawardena, P. Key, A. P. N. Singh, V. Balan, and G. Dejean, "Rethinking indoor wireless: Low power, low frequency, full duplex," Microsoft Research, Tech. Rep., 2009.

[7] "WARPLab framework." [Online]. Available: http://warp.rice.edu/trac/ wiki/WARPLab

[8] "RE07U-SM." [Online]. Available: http://www.l-com.com/item.aspx?id= 21447

[9] M. Duarte, A. Sabharwal, C. Dick, and R. Rao, "Beamforming in MISO systems: Empirical results and EVM-based analysis," IEEE Trans. Wireless Comm., vol. 9, no. 10, pp. 3214-1276, October 2010. 